\section{Successful use of antihistamines in severe hypereosinophilia}

\author{
Stefano Vallero, Anna Mondino, \\ Loredana Farinasso, Giulia Ansaldi, \\ Mirella Davitto, Ugo Ramenghi
}

Hematology Unit, Pediatric Department, Children's Hospital Regina Margherita, Turin, Italy

\section{Abstract}

Eosinophilia is common in childhood, and in most cases it is mild and of limited clinical relevance, being often secondary to allergy or infections. In rare cases, eosinophilia may be idiopathic or related to neoplastic aetiology. When severe and protracted, it can cause potentially irreversible organ or system damage, whose prevention is the first priority in the clinical management of hypereosinophilia. We describe the case of a patient with very severe eosinophilia, in whom antihistamines proved to be effective and safe in contributing to the eosinophil count normalization, thus avoiding the use of steroids until the hypothesis of an underlying neoplastic disorder was reasonably excluded.

\section{Introduction}

The finding of high eosinophil count is quite frequent in childhood, and in most cases it is mild and transient, but sometimes can be the first sign of a severe pathological condition. Hypereosinophilia is defined as a peripheral blood absolute eosinophil count (AEC) higher than $0.6 \times 109 / \mathrm{L}\left(0.7 \times 10^{9} / \mathrm{L}\right.$ in neonates $) .1,2$

The degree of eosinophilia can be further categorized into mild (AEC $0.6-1.5 \times 109 / \mathrm{L}$ ), moderate (AEC $1.5-5 \times 109 / \mathrm{L}$ ), or severe (AEC $\left.>5 \times 10^{9} / \mathrm{L}\right) .^{3}$

Eosinophilia can be primary (idiopathic) or secondary to allergy, infections, connective tissue disease, or cancer. While mild eosinophilia is frequent in childhood, being most commonly related to allergy, ${ }_{1}$ moderate and severe eosinophilia is rare. Usually, children with allergic diathesis show mild to moderate eosinophilia, with AEC rarely exceeding $1.0-2.0 \times 109 / \mathrm{L}$. Higher AEC may be the uncommon yet possible first sign of neoplastic disease, sometimes being the result of a clonal eosinophilic proliferation or secondary to other neoplastic diseases (lymphoproliferative or myeloproliferative diseases, and even solid tumors). ${ }^{4} \mathrm{~A}$ diagnosis of hypereosinophilic syndrome (HES) should be considered when eosinophilia is sustained $(>1.5 \times 109 / \mathrm{L})$ and protracted with evidence of target organ damage. ${ }^{3} \mathrm{HES}$ is a myeloproliferative disorder with multi-organ systemic involvement, that is frequently associated with peculiar acquired genetic aberrations (FIP1L1-PDGFRA fusion gene). 4 The therapy of HES is challenging and encompasses the use of tyrosine-kinase receptor inhibitors (e.g. imatinib) and sometimes allogenic hematopoietic stem cell transplantation. 5

Severe or protracted eosinophilia may induce organ damage due to the toxic action of pro-inflammatory cytokines released by the eosinophils. The target organs and systems most frequently involved are the heart, the nervous system, and the skin. Involvement of either the heart or the central nervous system is responsible for significant morbidity and mortality. 6,7

Corticosteroids are useful in lowering the AEC, but their use might mislead and delay the diagnosis in patients in whom a malignant hemopathy underlies eosinophilia. Ideally, the use of steroids in patients with eosinophilia should be started only when the diagnostic process has led to a reasonable exclusion of an underlying neoplastic disease.

\section{Case Report}

A 7-year-old boy was evaluated for malaise, anorexia and recurrent fever. In the absence of organomegaly, lymphadenopathy or other signs of lymphoproliferative disease, a complete blood count evidenced isolated very severe hypereosinophilia, (white blood cells $70 \times 109 / \mathrm{L}$, with $80 \%$ eosinophils), with normal haemoglobin and platelets. The patient, as well as his parents, reported a history of mild allergy.

Peripheral blood smear showed a huge number of morphologically normal eosinophils, without signs of lymphoproliferative disease or myelodysplasia.

Total immunoglobulin E (IgE) was elevated $(233 \mathrm{UI} / \mathrm{mL}$ n.v. < 70) and the search for specific IgE - FAST - resulted positive for dermatophagoides pteronyssinus, cat epithelium, alternaria and parietaria, egg and milk; prick tests for food and inhalants were negative.

Due to the very high eosinophil count, antihistamines were administered immediately (cetirizine, $5 \mathrm{mg} / \mathrm{day}$ ), in order to try and reduce hypereosinophilia while avoiding the use of corticosteroids.

In order to exclude infections, the following exams were performed: Toxocara and Toxoplasma serology, Epstein-Barr virus serology, throat swab, stool and urine culture, Mantoux inthradermoreaction, stool parasitological exam, the search for Aspergillum anti-
Correspondence: Stefano Vallero, Hematology Unit, Pediatric Department, Children's Hospital Regina Margherita, Piazza Polonia 94, Torino, Italy.

Tel. +39.011 .3135259 - Fax: +39.011 .3135382 .

E-mail: stefano.vallero@gmail.com

Key words: hypereosinophilia, allergy, antihistamines, corticosteroids.

Conflict of interests: the authors report no potential conflict of interests.

Received for publication: 27 January 2012. Accepted for publication: 12 February 2012.

This work is licensed under a Creative Commons Attribution NonCommercial 3.0 License (CC BYNC 3.0).

(C) Copyright S. Vallero et al., 2012

Licensee PAGEPress, Italy

Pediatric Reports 2012; 4:e26

doi:10.4081/pr.2012.e26

gen, Widal Wright serology, oculistic examination. All exams resulted negative.

Autoimmunity (Coombs test, anti-nucleus and anti DNA antibody, celiac disease screening and HLA DQ2 and DQ8 search) was negative.

Chest X-ray and abdominal ultrasound were normal.

A bone marrow aspirate was performed, but both morphology and flow cytometry disclosed abundant eosinophils with no leukemic cells or myelodysplasia. Chromosomal rearrangements commonly associated with myeloid and lymphoblastic acute leukemia were negative. Karyotype was $46, \mathrm{XY}$. The rearrangement FIP1L1-PDGFRA, typical of HES, was negative both on peripheral blood and on bone marrow. The search for WT-1 (Wilms tumor gene) gene copy number alteration, reported to be high in HES, ${ }^{8}$ was negative.

Within less then 2 weeks of treatment with antihistamines, hypereosinophilia improved significantly, and a normalizazion of AEC was obtained in 12 days $(0.46 \times 109 / \mathrm{L})$.

After eosinophil count normalization, the patient was discharged and followed-up with periodical clinico-hematological controls. Electrocardiogram and cadiac ultrasound were repeated weekly and never showed cardiac functional damage. Hepatic aminotransferases and creatinine were always normal. No skin rash was noticed. Neither intestinal bleeding nor diarrhea was reported.

Fifteen months later, in the absence of newer signs or symptoms, eosinophils raised again severely (AEC $45.4 \times 109 / \mathrm{L}$ ). Blood smear and flow cytometry showed normal morphology and immunophenotype. IgE was found to be elevated again $(156 \mathrm{UL} / \mathrm{mL})$. 


\section{MODERATE OR SEVERE HYPEREOSINOPHILIA}

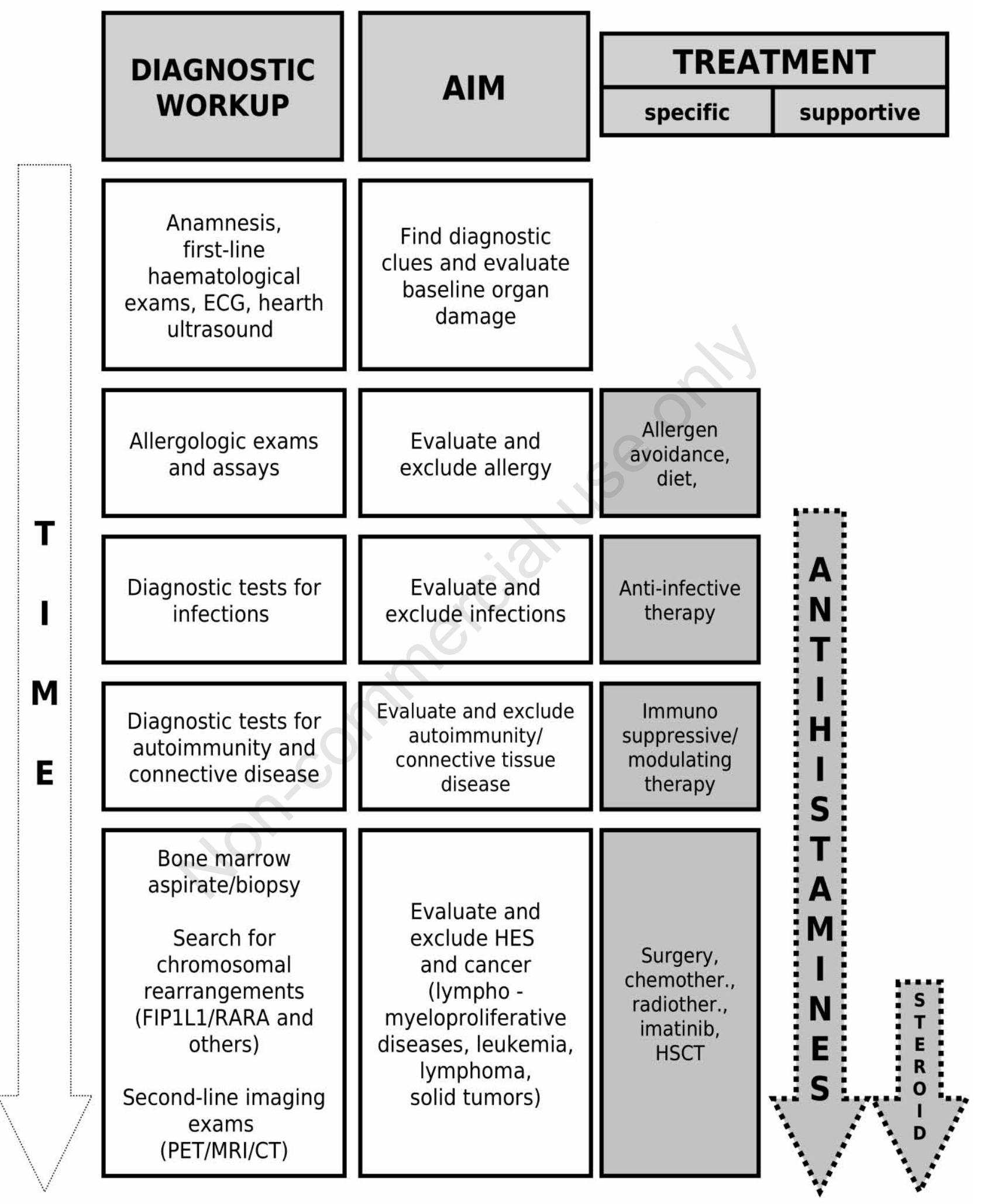

Figure 2. Diagnostic work-up and treatment (specific and supportive) as proposed by the Authors. Antihistamines may be started safely and promptly to prevent organ damage, the best timing being after completing allergy exams. On the contrary, steroids should not be administered until a diagnosis of hypereosinophilic syndrome or neoplastic disease is ruled out. 

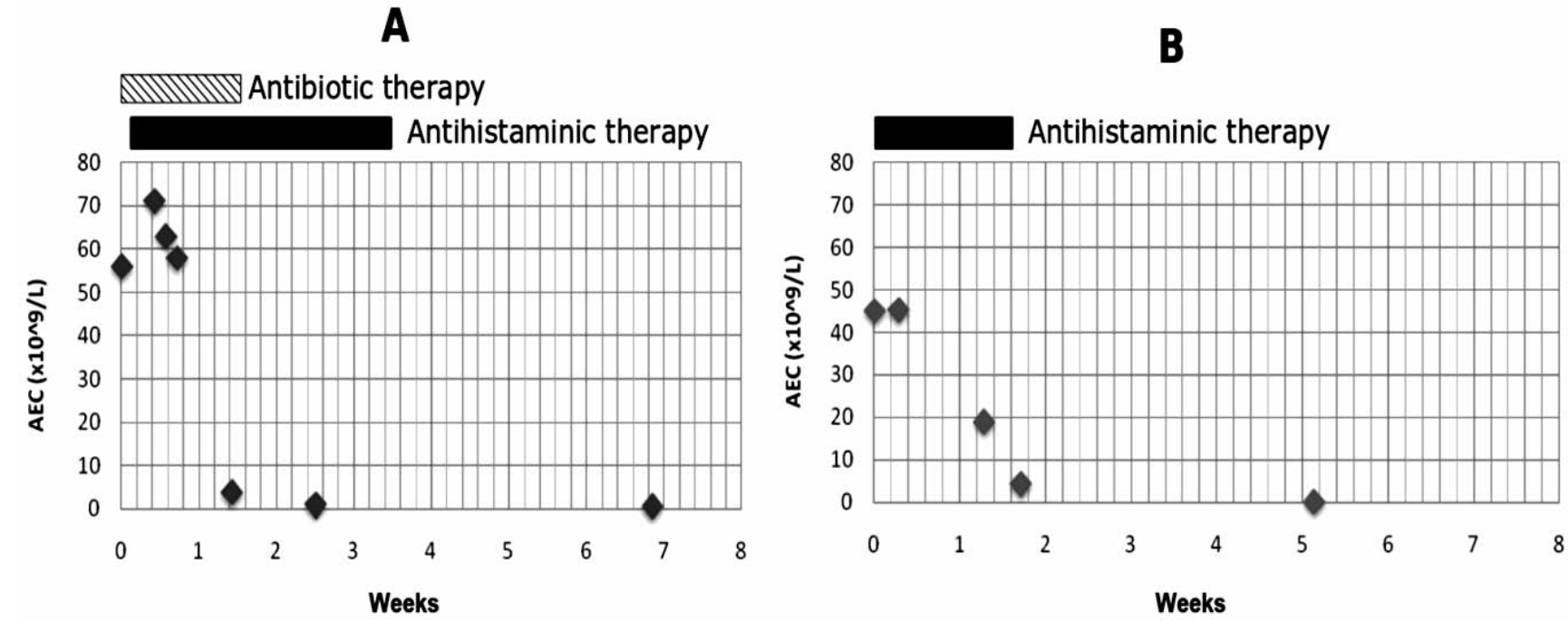

Figure 1. Absolute eosinophil count trend and concomitant therapy during the first (A) and the second hospitalization (B).

The patient was immediately treated with antihistamines again, and AEC lowered to normal values in 3 weeks (Figure 1). An allergenfree diet was started; the patient has been followed up for 12 months and at the time of writing his AEC was normal.

\section{Discussion and Conclusions}

Allergy is among the most frequent cause underlying eosinophilia, especially in children. The typical clinical picture in allergic patients with eosinophilia is mild-to-moderate AEC elevation in presence of clinical manifestations of allergic disease (rhinitis, conjunctivitis, skin rash, eczema, urticaria, etc.) without relevant signs of systemic organ or tissue involvement.

In our patient very severe eosinophilia (highest AEC $70 \times 109 / \mathrm{L}$ ) was associated with mild systemic symptoms with fever and malaise, but no typical signs of allergic diathesis. Clinical priorities for physicians were to exclude a neoplastic etiology or HES, on one hand, and to prevent potential system and organ damage, on the other.

The peculiarity of our patient was that the extremely high AEC was secondary to allergy. At presentation, we can hypothesize that AEC was boosted by an unidentified viral infection, that was probably the cause of fever, but this is just speculative.

A wide panel of clinical, haematological and imaging exams reasonably excluded an underlying neoplastic disease. The translocation FIP1L1-PGDFRA was negative on bone marrow aspirate; 4 furthermore, normal WT-1 quantitative analysis, ${ }^{8}$ the presence of positivity to allergogenic tests and the rapid and complete normalization of AEC with antihistaminic therapy led us to consider HES and neoplasia as improbable aetiologies.

Very high levels of eosinophils are a possible finding in patient with allergy, especially when associated with other pro-inflammatory events such as viral or bacterial infections, and very severe eosinophilia is not invariably associated with myeloproliferative hypereosinophilic syndrome or other neoplastic disease.

Eosinophil morphology, normality of cell blood count and other hematopoietic series, response to antihistaminic treatment and positivity of allergogenic assays can ease the diagnosis of allergic eosinophilia. Hematologic and imaging exams can help exclude the presence of neoplastic lesions that could indirectly lead the eosinophilic proliferation.

The surveillance, prevention and early treatment of systemic and organ damage due to sustained and prolonged eosinophilia remains one of the mainstays in the care of eosinophilic patients and must accompany all steps of the diagnostic and therapeutic process. The effectiveness of antihistamines in severe hypereosinophilia is not described in literature, but our case supports the hypothesis that antihistaminic therapy may sometimes be sufficient to reduce $\mathrm{AEC}$, and that potentially it can be used as a first-line therapy in a subset of patients with severe eosinophilia. The use of second-generation antihistamines at conventional dosage is recommended, due to the low incidence of collateral effect, even when used for long periods. The use of steroidal drugs should be avoided in the diagnostic phase, and limited to those patients in whom a complete diagnostic process has led to a reasonable exclusion of an underlying neoplastic disease (Figure 2).

\section{References}

1. Rothenberg ME, Hogan SP. The eosinophil. Annu Rev Immunol 2006;24:147-74.

2. Patel L, Garvey B, Arnon S, Roberts IA. Eosinophilia in newborn infants. Acta Paediatr 1994;83:797-801.

3. Brito-Babapulle F. The eosinophilias, including the idiopathic hypereosinophilic syndrome. Br J Haematol 2003;121:203-23.

4. Tefferi A, Gilliland D. Oncogenes in myeloproliferative disorders. Cell Cycle 2007;6: 550-66.

5. Klion AD, Bochner BS, Gleich GJ, et al. Approaches to the treatment of hypereosinophilic syndromes: a workshop summary report. J All Clin Immunol 2006;117: 1292-302.

6. Katz HT, Haque SJ, Hsieh FH. Pediatric hypereosinophilic syndrome (HES) differs from adult HES. J Pediatr 2005;146:134-6.

7. Cools J, DeAngelo DJ, Gotlib J, et al. A tyrosine kinase created by fusion of the PDGFRA and FIP1L1 genes as a therapeutic target of imatinib in idiopathic hypereosinophilic syndrome. New Engl J Med 2003;348:1201-14.

8. Cilloni D, Messa F, Martinelli G, et al. WT1 transcript amount discriminates secondary or reactive eosinophilia from idiopathic hypereosinophilic syndrome or chronic eosinophilic leukemia. Leukemia 2007;21: 1442-50. 